\title{
Multi-Agent-Based Simulation for Formation of Institutions on Socially Constructed Facts
}

\author{
Takashi Hashimoto ${ }^{1}$ and Susumu Egashira ${ }^{2}$ \\ 1 School of Knowledge Science, \\ Japan Advanced Institute of Science and Technology (JAIST), \\ Nomi, Ishikawa, 923-1292, Japan \\ hash@jaist.ac.jp, http://www.jaist.ac.jp/ hash/index-e.html \\ 2 Otaru University of Commerce, \\ 3-5-21, Midori, Otaru, 047-8501, Japan \\ susumue@ba2.so-net.ne.jp, http://www.res.otaru-uc.ac.jp/ ^egashira/
}

\begin{abstract}
In human societies, facts are constructed through social consensus. Here, the formation of social institutions in such a society is studied using a multi-agent-based simulation. Institutions are formed through communications among members, and the effects of errors in communication on the formation of institutions are investigated. Our results show that the institution is established when information suppliers frequently make errors in their information interpretation. We propose here that there is a phase transition in the error rate of the information suppliers in the formation of institutions.
\end{abstract}

\section{Introduction}

In the present study, we examine the formation of social institutions in a society using a multi-agent simulation. In particular, we investigate how errors in communication among members of the society affect the formation of institutions, when the "facts" emerge from interactions between the members.

One of the remarkable features of humans is that we live in societies and construct cultures. Here, we define culture as dominant modes of action and thought that are inherited through non-genetic mechanisms and are retained in a group of organisms. Among animals that form societies and cultures, human cultures are distinguished by their arbitrariness[1]. In animal societies, most cultures are related to survival and reproduction, such as methods of food utilisation and avoidance of enemies, while in human societies, a particular method or form is selected from among possible arbitrary options and regarded as formal, e.g., funeral rites and costumes used in rituals. This means that formality and correctness of codes of conduct and ethics in a society, such as morals and justice, are decided both unconsciously and unintentionally by members of that society.

Further, facts are sometimes determined by social consensus. For example, firms estimate their performance according to an accounting system. The measure of the estimation, such as the depreciation rate, is determined politically by agreement among public organisations. In a sense, the evaluation of a firm 
is largely dependent on the social consensus, and if a criterion is changed, the value of the firm is also changed without any accompanying physical change[2]. That is, facts about the firm's performance are constructed based on the social consensus. This character of facts is called "social construction of facts". It is discussed that our reality also depends on the social construction $[3,4]$.

Systems that regulate our behaviour, such as the accounting system and laws, and that form the basis of our thoughts, such as customs and ethics, are called social institutions. Veblen[5] defined institutions as "settled habits of thought common to the generality of men". Social institutions are often made up through communication. People living in a complex society, in which little firsthand or direct information about various events is obtained, make their decisions according to information obtained from others. However, it is logically impossible to confirm the correctness of the information gained, because the confirmation of certain information requires additional information, which also requires additional information for confirmation, and so on. We called this character the "fundamental imperfection of information" and concluded that institutions work effectively to economise the cost for each person to confirm the correctness of information by believing the institutional systems established in the society[6].

Multi-agent-based simulations have been used to study the formation of institutions and norms[7-10]. These studies barely consider "fundamentally imperfect information" and the development of the agents' cognitive frameworks (world views or ways of thought) through interactions with others. Our previous work[11] showed that an institution as an ordered cognitive framework is formed as a result of social learning, such as the imitation of others' superficial actions and the continuous revisions of internal cognitive frameworks. However, in these studies, the social construction of facts and errors in the communication process are not considered. Thus, the present study was performed to investigate how institutions are formed when facts or partial facts are determined socially and how errors in information interpretation affect the formation of institutions.

Here, we suppose that people obtain information about some objective situations to be dealt with through communication, interpret it and act according to the interpretations. A typical example is the stock market where many investors make decisions about investments in stocks of various firms according to information supplied by securities companies and rating agencies. How their decisions are evaluated, i.e., their profit or loss, depends largely on the actions of all investors. Keynes[12] likened this situation to a "beauty contest" in which not only does the prize go to the person who receives the most votes but in which those who vote for the winner also benefit. Thus, it is thought that the validity of investors' decisions is partially socially constructed. To understand firms' performances, the investors must not only adequately select the information suppliers but also correctively interpret the information supplied. Note that the objective phenomena to be modelled in this paper are not limited to economic activities. As mentioned above, there are many activities in which codes of conduct and ways of thinking are formed through communication and that affect our activities. 
This paper is organised as follows: In Sec. 2, a multi-agent model is introduced. In Sec. 3, simulation results using the model are described. We discuss the results from the viewpoint of the formation of institutions in Sec. 4. The conclusions are presented in Sec. 5 .

\section{$2 \quad$ Model}

We incorporated incorporate the social construction of facts and errors in information interpretation into our previous model[11]. The present model consists of two types of agents, information suppliers and information receivers, and objective situations with which the information receivers should deal. The information flows from the objective situations to the receivers through the suppliers, as illustrated schematically in Fig.1. An event sequence from setting an objective situation to evaluating the receivers' decision is called one turn.

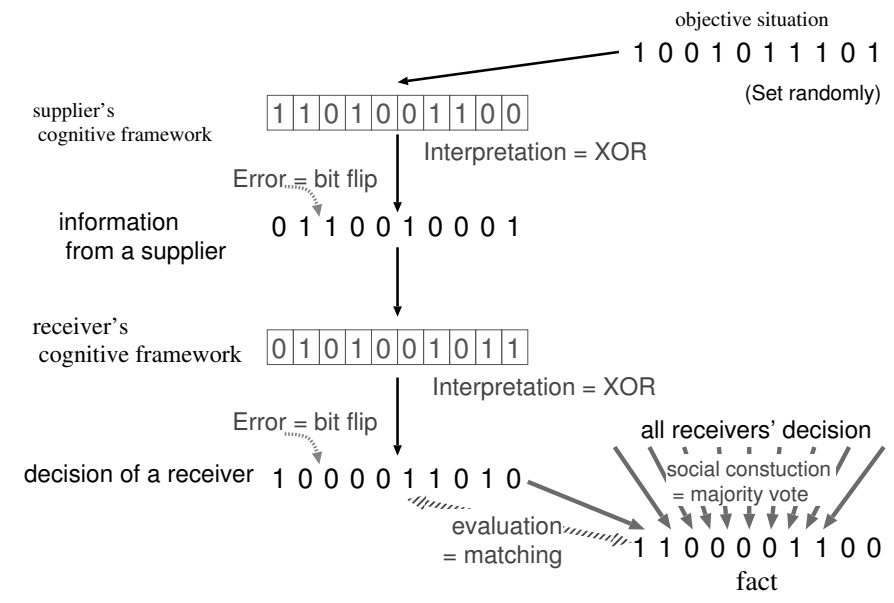

Fig. 1. The Information flow from an object to a supplier and to a receiver.

Each agent has its own cognitive framework for interpreting information. The framework is expressed by a bit string $\boldsymbol{f}^{S}=\left(f_{1}^{S}, f_{2}^{S}, \cdots, f_{L}^{S}\right)$ for a supplier and $f^{R}=\left(f_{1}^{R}, f_{2}^{R}, \cdots, f_{L}^{R}\right)$ for a receiver. Only the information receivers are aligned on a 2-dimensional $W \times W$ cell-plane with a periodic boundary.

The information suppliers observe the objective situations. One objective situation consists of $L$ figures, each figure has two states: 0 or 1 . The objective situation is expressed by a vector $\boldsymbol{O}=\left(\mathrm{O}_{1}, \mathrm{O}_{2}, \cdots, O_{L}\right)$. Each figure corresponds to each element of the frameworks, $f^{S}$ 's and $f^{R}$ 's. The objective situation is randomly generated at the beginning of each turn.

The information suppliers interpret the objective situations. The way of interpretation is implemented by the exclusive or (XOR) bit operation, defined 
as

$$
\operatorname{XOR}(x, y)=\left\{\begin{array}{l}
0(x=y) \\
1(x \neq y)
\end{array} .\right.
$$

Errors in the interpretation are implemented by the bit flip operation, defined as

$$
F \operatorname{lip}(x)=\left\{\begin{array}{l}
0(x=1) \\
1(x=0)
\end{array} .\right.
$$

The error function, Flip, is operated randomly on each bit according to the suppliers' error rate $\varepsilon^{S}$ per bit. Thus, a supplier's interpretation, $\boldsymbol{I}^{S}=\left(I_{1}^{S}, I_{2}^{S}, \cdots, I_{L}^{S}\right)$, of information about an objective situation is expressed as:

$$
I_{i}^{S}=\operatorname{Flip}\left(X O R\left(O_{i}, f_{i}^{S}\right)\right), \quad(i=1 \sim L) .
$$

Each information receiver, located on a $2 \mathrm{D}$ plane of size $W \times W$, adopts a supplier as the source of information about the objective situations. A receiver obtains information, $\boldsymbol{I}^{S}$, from the adopted supplier and makes its own interpretation, $\boldsymbol{I}^{R}$, using its cognitive framework, $\boldsymbol{f}^{R}$, in the same manner as the information supplier:

$$
I_{i}^{R}=\operatorname{Flip}\left(X O R\left(I_{i}^{S}, f_{i}^{R}\right)\right), \quad(i=1 \sim L) .
$$

The error rate of the receivers is denoted by $\varepsilon^{R}$. The receivers make decisions based on their interpretation. In this paper, for simplicity, the decision is identified with the interpretation.

The receivers' decisions are evaluated in terms of facts, denoted by $\boldsymbol{F}=$ $\left(F_{1}, F_{2}, \cdots, F_{L}\right)$, which are socially constructed through the majority vote,

$$
F_{i}=\operatorname{Majority}\left(I_{i}^{R}\right)=\left\{\begin{array}{ll}
1 & \left(\sum_{\text {all receivers }} I_{i}^{R}>W^{2} / 2\right) \\
0 & \text { (otherwise) }
\end{array} .\right.
$$

That is, the fact for $i$ th bit is 1 if more than half of the receivers interpret it as 1 , and vice versa. Each receiver scores the number of bits in its own interpretation, $\boldsymbol{I}^{R}$, that match the fact, $\boldsymbol{F}$. Thus, the score of a receiver, denoted by $P$, is:

$$
P=\sum_{i=1}^{L}\left(1-\operatorname{XOR}\left(I_{i}^{R}, F_{i}\right)\right)
$$

After evaluation, each receiver compares its score with those of the eight neighbouring receivers. If a receiver has the lowest score alone, then a randomly selected element in its cognitive framework is altered; otherwise nothing happens. The lowest scored agent also changes its information supplier, adopting the supplier adopted by the best receiver among its neighbours. If more than one receiver has the best score in its neighbours, one is selected at random. That is, the locally worst receiver imitates the selection of supplier - i.e., the externally observable behaviour - of the locally best receiver and internally searches a better framework in a trial-and-error manner, which is the least smart and memory-less learning method. 


\section{Simulation Results}

We report the results of simulation using the model described above. The conditions for the simulation were as follows. The size of the information receivers' plane was $W=21$, and the numbers of receivers and of information suppliers were both 441 . The length of the bit strings for the objective situations, the frameworks and the fact was $L=10$. The initial cognitive frameworks were set randomly. While the receivers revised their frameworks according to the score, $P$, the suppliers did not change from their initial framework. Varying the error rates, $\varepsilon^{S}$ and $\varepsilon^{R}$, we assess the effect of errors on the formation of institutions.

\subsection{Erroneous Interpretation by Suppliers}

To assess the effects of the suppliers' interpretation error on the formation of institutions, we conduct experiments in which only the suppliers make errors. The error rates are $\varepsilon^{S}=1 E-5,1 E-4,0.001,0.01,0.1$ and 0 ; and $\varepsilon^{R}=0$.

We observed how the receivers' selection of suppliers changed over time. A group in which the receivers adopted the same supplier is called a cluster. The size of the $k$ th cluster, denoted by $C_{k}$, is the number of receivers in the cluster. The graph shown in Fig. 2(a) shows the dynamics of the size of the largest cluster, $C$, for various values of $\varepsilon^{S}$. Only in the case of $\varepsilon^{S}=0.1$, the cluster size expanded rapidly and reached the maximum, $C_{\max }=W^{2}=441$. Clusters hardly developed for other values of the error rate.

The degree of (dis)accordance between the cognitive frameworks of the receivers is measured by the Hamming distance between two receivers' frameworks

$$
\operatorname{dist}\left(r, r^{\prime}\right)=\sum_{i=1}^{L}\left|f_{i}^{r}-f_{i}^{r^{\prime}}\right|
$$

where $r$ and $r^{\prime}$ represent two receivers and $|\cdot|$ is the absolute value. The average distance in the $k$ th cluster,

$$
D_{k}=\frac{1}{2 C_{k}} \sum \operatorname{dist}\left(r, r^{\prime}\right)
$$

is a measure of the (dis)accord of the framework in the cluster, where the sum is taken over all receivers, $r$ and $r^{\prime}$, in the $k$ th clusters.

Figure 2(b) shows the dynamics of the average distance in all clusters,

$$
D_{\text {ave }}=\frac{1}{W^{2}} \sum_{k} C_{k} D_{k} .
$$

Except in the case of $\varepsilon^{S}=0.1$, the average distance converges to a value that depends in a straightforward manner on the error rate. Specifically, larger error rate were associated with grater distance. In the case of $\varepsilon^{S}=0.1$, following the rapid growth of the largest cluster (around 7,000th turn), the receivers' frameworks begin to show accordance (around 8,000th turn) and finally become completely common to all the receivers $\left(D_{\text {ave }}=0\right.$, around 30,000 th turn). 

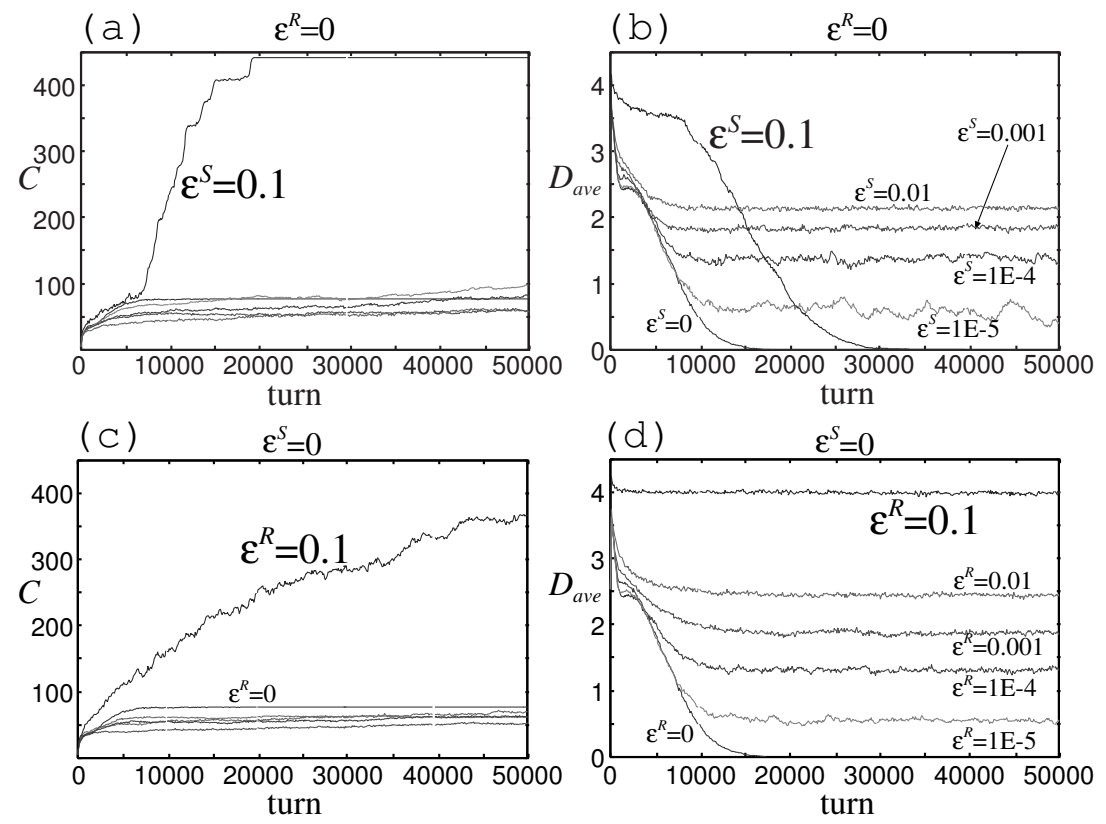

Fig. 2. The dynamics of the largest cluster size, $C$ ((a) and (c)), and the average distance in clusters, $D_{\text {ave }}((\mathrm{b})$ and $(\mathrm{d}))$, when the suppliers made errors in their interpretation process $((\mathrm{a})$ and $(\mathrm{b}))$, and when the receivers made such errors $((\mathrm{c})$ and $(\mathrm{d}))$. The $x$-axis is the turn. The error rates are $\varepsilon^{S}=1 E-5,1 E-4,0.001,0.01,0.1$, and 0 ; and $\varepsilon^{R}=0$ in (a) and (b); $\varepsilon^{R}=1 E-5,1 E-4,0.001,0.01,0.1$, and 0 ; and $\varepsilon^{S}=0$ in (c) and (d). All results are ensemble averages over 10 runs for each point.

\subsection{Erroneous Interpretation by Receivers}

The situation differs from the previous case when only the receivers make errors $\left(\varepsilon^{R}=1 E-5,1 E-4,0.001,0.01,0.1\right.$, and 0 ; and $\left.\varepsilon^{S}=0\right)$. The dynamics of the largest cluster size, depicted in Fig. 2(c), is similar to the case of supplier's error, Fig. 2(a). Only when $\varepsilon^{R}=0.1$, the largest error rate in the present experiments, a cluster expanded. However, the speed of growth of the cluster was much slower than in the case of suppliers' error. The average distance did not decrease at all for $\varepsilon^{R}=0.1$, as shown in Fig. 2(d).

\subsection{Social and Physical Facts}

In our society, not all facts are constructed fully socially, but some are determined physically or externally. How a fact is determined socially or physically is a matter of gradient. Therefore, in addition to social construction, we incorporated the physical determination of facts into our model by identifying the facts with the objective situation. That is, if the $i$ th bit of a fact is determined physically, 
then $F_{i}=O_{i}$. The degree of social construction is parameterised by $S c=L_{s} / L$, where $L_{s}$ is the number of bits constructed by the majority vote, (5). In this study, each bit was fixed to either a social or physical fact according to the parameter $S c$.

Figure 3 shows the average distances in clusters at the stationary states for various values of $S c$ for both cases of suppliers' (Fig. 3(a)) and receivers' errors (Fig. 3(b)). In the case of $\varepsilon^{S}=0.1, \varepsilon^{R}=0$ and $S c=1.0$, the receivers' frameworks come to complete accordance, as described in the previous section. This accordance is broken when no more than one bit is determined physically, i.e., $S c<1.0$. The mixture of social and physical facts affects on the formation of institutions only for $\varepsilon^{S}=0.1, \varepsilon^{R}=0$. The other lines in Fig. 3 are virtually flat for all values of Sc.

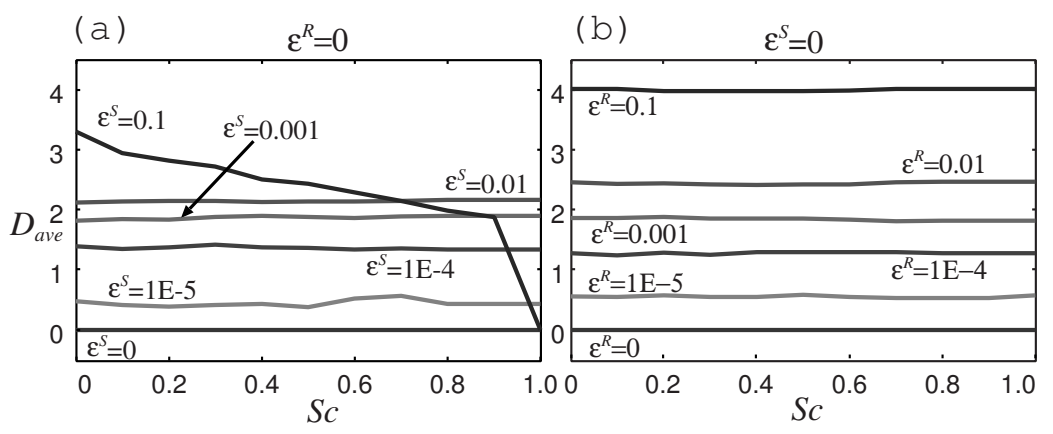

Fig. 3. Effects of the mixture of social and physical facts on (dis)accordance of cognitive frameworks. The $x$-axis shows the ratio of social construction in facts $(S c)$, and the facts are fully socially constructed at the right edge, $\mathrm{Sc}=1.0$. The $y$-axis shows the average distance in clusters. (a) The suppliers make errors, $\varepsilon^{R}=0$. (b) The receivers make errors, $\varepsilon^{S}=0$. All results are ensemble averages over 10 runs for each point.

\section{Discussion}

\subsection{Superficial and Cognitive Regularity}

We showed that the effects of errors at an error rate of 0.1 on cluster formation and accordance of frameworks were different from the other error rates. Further, the effects also differed between suppliers' and the receivers' errors. The results for the social construction of facts are summarised in Table 1.

At an error rate of 0.1 , the relative cluster size is 1 for both the suppliers' and the receivers' errors. This situation represents the formation of superficial regularity. Large-scale errors in the information supplied or of the receivers' interpretation cause large-scale fluctuations in the receivers' scores, which promotes the receivers' revision of suppliers. As the change is based on imitation of 
Table 1. Effects of errors on cluster formation and the accordance of frameworks. The relative cluster size is the ratio of cluster size to all receivers.

\begin{tabular}{cc|cc}
\hline error by & error rate & the relative cluster size & distance in clusters \\
\hline \hline suppliers' & $<0.1$ & $0.1 \sim 0.2$ & $0.5 \sim 2.5$ \\
& 0.1 & 1 & 0 \\
\hline receivers' & $<0.1$ & $0.1 \sim 0.2$ & $0.5 \sim 2.5$ \\
& 0.1 & 1 & $\sim 4.0$ \\
\hline
\end{tabular}

the externally observable behaviours of others, i.e., selection of the information supplier, the selections are finally canalised to one supplier.

For suppliers' error, there is also regularity in the cognitive frameworks, $D_{\text {ave }}=0$, when the error rate is $\varepsilon^{S}=0.1$. Once all receivers obtain information from only one supplier, the interpretation error by the supplier does not matter, as the facts are constructed by the receivers themselves through a majority vote based on the uniform information in the society. Any receiver with a different framework from the majority must revise its framework to conform to the majority. Thus, the cognitive frameworks are built up until they are common to all receivers. This situation provides individuals in the society with consistency/regularity in their world views and has a self-enforcement function. Accordingly, we consider this situation the formation/establishment of a (cognitive) institution.

In contrast, frequent receiver error $\left(\varepsilon^{R}=0.1\right)$ does not result in concordance of the cognitive frameworks, i.e., $D_{\text {ave }} \cong 4$.0. The error prevents them achieving a good score and they keep changing their framework forever.

When the fact is partly determined physically, the formation of an institution cannot be completed. There remains diversity in the cognitive framework, $D_{\text {ave }}>0$, as shown in Fig. 3. Suppliers' interpretation errors about the physically determined facts induce the receivers to revise their frameworks. With regard to physical facts, the revisions keep occurring as the receivers are not forced to come into accordance with the majority and frequent errors by the suppliers weaken enforcement upon receivers to conform to the same framework as the suppliers ${ }^{3}$.

\subsection{Phase Transition at Error Threshold}

At an error rate of 0.1 per bit, the agents always make errors in interpretation as the frameworks are 10 bits in length. This value corresponds to the error threshold, $\varepsilon_{t h}=1 / L$, which was proposed by Eigen[13] as the critical accuracy of information copy in the context of the origin of life. He showed that there was a phase transition at the critical value in the distribution of information entities $^{4}$.

\footnotetext{
${ }^{3}$ When there is no error, $\varepsilon^{S}=\varepsilon^{R}=0$, the enforcement works well, as shown in our previous study[11], and indicated in Fig. 3 for all cases of the mixture of facts.

${ }^{4}$ The information entities are genes in the context of the origin of life.
} 
Figure 4 shows thorough calculation of the dependence of the average distance, $D_{\text {ave }}$, on the suppliers' error rate, $\varepsilon^{S}$, and the degree of social construction, $S c$. The distance, $D_{\text {ave }}$, is an order parameter in this system, as complete accordance of the framework in clusters brings the distance $D_{a v e}=0$, and the random state $D_{\text {ave }}=L / 2$. The distance increases monotonically with the error rate from small values to $\varepsilon^{S} \cong 0.03$. It decreases for large values of $S c$ at $\varepsilon^{S}=0.03 \sim 0.1$. Especially, for $S c=1.0$, it falls abruptly to $D_{\text {ave }}=0$, i.e., complete order. This abrupt descend indicates full accordance of the cognitive framework, i.e., social order, established at $\varepsilon^{S}=0.1$. This result suggests a phase transition in the formation of institutions at the point of the error threshold of the suppliers' error, when facts are mainly constructed socially. In this graph, the change to $D_{\text {ave }}=0$ is not completely discontinuous but is smooth. This may be caused by the finiteness of the system.

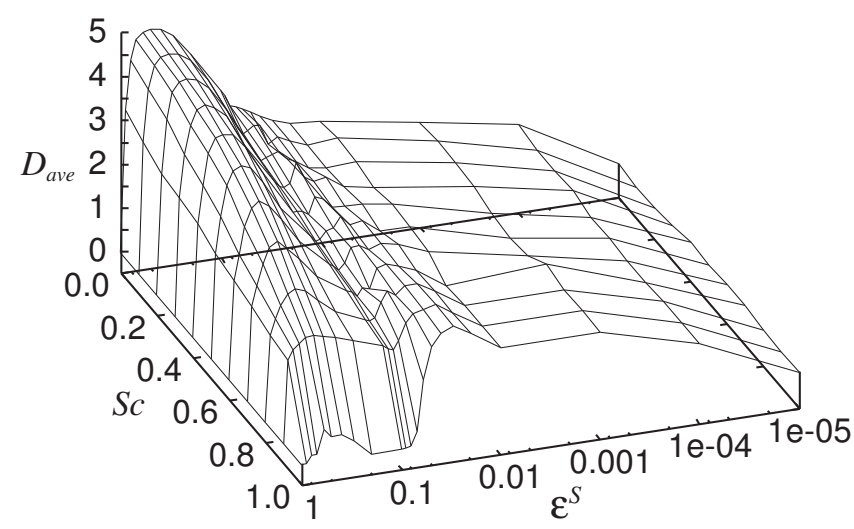

Fig. 4. Dependence of the average distance on the suppliers' error rate, $\varepsilon^{S}$ (log scale), and the degree of social construction, $S c$. These results are ensemble averages over 10 runs for each point.

\section{Conclusion}

Using a multi-agent-based simulation, in which agents are equipped with adaptive cognitive frameworks, we studied the formation of institutions when the facts are constructed through social consensus.

Our simulation results suggested the following conditions for the formation of cognitive institutions, the ordered state of the cognitive framework in a society: 1) the information suppliers frequently make interpretation errors; 2) the information receivers seldom make interpretation errors; and 3) facts are constructed 
through social consensus. It was also suggested that there is a phase transition at the error threshold of the suppliers' error in the formation of institutions.

The present system remains to be improved in regard to several points. The model of agents is very simple and static, and we should therefore test how the results are reproduced with a more dynamic agent model, such as that of a cognitive individual with internal dynamics[14]. Another point is the introduction of a temporal correlation between objective situations and to let the agents predict the future situation. This is also possible by using the individual model with internal dynamics. Future studies should also compare the suggested conditions to empirical evidence. Of course, while there are many difficulties in direct comparisons, political elections would be a possibility.

Acknowledgments The authors thank Prof. Sawabe for valuable discussions and the anonymous reviewers for improving the paper. This work was supported in part by a Grant-in-Aid for Scientific Research (No.17651088) from the Ministry of Education, Culture, Sports, Science and Technology of Japan and by Japan Society for the Promotion of Science.

\section{References}

1. Merker, B.: From ape culture to ritual culture and language via vocal learning for song: The singular path to human uniqueness. S. Malloch and C. Trevarthen (Eds.), Communicative Musicality, Oxford University Press (forthcoming)

2. Sawabe, N: private communication

3. Berger, P.L., Luckmann, T.: The Social Construction of Reality: A Treatise in the Sociology of Knowledge, Anchor (1967)

4. Searle, J.R.: The Construction of Social Reality, Free Press (1995)

5. Veblen, T.B.: The Place of Science in Modern Civilisation and Other Essays, Huebsch (1919)

6. Egashira, H., Hashimoto, T.: The formation of common norms on the assumption of 'fundamentally' imperfect information. R. Conte, C. Dellarocas (Eds.), Social Order in Multiagent Systems, Kluwer (2001)

7. Conte R., Castelfranchi C.: Cognitive and Social Action, University College London Press (1995)

8. Epstein J.M., Axtell R.: Growing Artificial Societies, MIT Press (1996)

9. Conte R., Dellarocas C. (Eds.): Social Order in Multiagent Systems, Kluwer (2001)

10. Felix F., Polani D., Uthmann T., Modelling the emergence of possession norms using memes. Journal of Artificial Societies and Social Simulation 4(4) (2001)

11. Hashimoto, T., Egashira, S.: Formation of social norms in communicating agents with cognitive frameworks. Journal of Systems Science and Complexity 14 (2001) $54-74$

12. Keynes, J.M.: The General Theory of Employment, Interest, and Money, Macmillan Cambridge University Press (1936)

13. Eigen, M.: Selforganization of matter and evolution of biological macromolecules. Naturwissenschaften 58 (1971) 465-523

14. Sato, T., Hashimoto, T.: Dynamic social simulation with multi-agents having internal dynamics. K. Hashida, K. Nitta (Ed.) New Frontiers in Artificial Intelligence, Springer (forthcoming) 